\title{
Mn-Doped ZnSe Quantum Dots as Fluorimetric Mercury Sensor
}

\author{
Sundararajan Parani, Ncediwe Tsolekile, \\ Bambesiwe M.M. May, Kannaiyan Pandian and \\ Oluwatobi S. Oluwafemi
}

Additional information is available at the end of the chapter

http://dx.doi.org/10.5772/intechopen.70669

\begin{abstract}
Quantum dots (QDs), because of their exciting optical properties, have been explored as alternative fluorescent sensors to conventional organic fluorophores which are routinely employed for the detection of various analytes via fluorometry. QD probes can detect toxic metal ions, anions, organic molecules with good selectivity and sensitivity. This chapter investigates the synthesis of Mn-doped $\mathrm{ZnSe}$ QDs using nucleation-doping strategy. The as-synthesized QDs were characterized by various analytical tools such as ultravioletvisible (UV-vis) absorption, photoluminescence (PL) spectroscopy, X-ray diffractometry (XRD) and transmission electron microscopy (TEM). It was found that Mn doping of QDs significantly increases the PL intensity. The PL of the resulting QDs was examined in the presence of different metal ions to check its selective response. Among the various metal ions, $\mathrm{Hg}^{2+}$ exhibits a drastic quenching of the QD's emission intensity. A Stern-Volmer plot of $\left[\mathrm{Hg}^{2+}\right]$ sensing using the as-synthesized QDs showed linearity in the range of $0-30 \times 10^{-6}$ $\mathrm{ML}^{-1}$ with the regression coefficient $\mathrm{R}^{2}=0.99$. The detection limit was found to be $6.63 \times$ $10^{-7} \mathrm{ML}^{-1}$. Thus, the present Mn-doped ZnSe QDs represent a simple, non-toxic fluorescent probe for the qualitative and quantitative detection of mercury ions in aqueous samples.
\end{abstract}

Keywords: quantum dots, ZnSe, fluorimetry, doping, heavy metal detection

\section{Introduction}

Continuous and high-concentration exposure of heavy metals can cause various physiological and biochemical problems to the environment and human being. Thus, the detection of these harmful metal ions has become very important from industrial, environmental and biological point of view. This is a challenging subject for analytical chemists due to the 
sensitivity, detection limits and acceptable toxicity levels set by global regulation bodies [1, 2]. In addition, similar chemistry of heavy metals is fastidious with respect to selectivity of the determination method. A variety of standardized analytical methods for the detection of metal ions are available. However, only some of them have found application in routine analysis. Recommended procedures for the detection of heavy metals in water samples include photometric methods, flame or graphite furnace atomic absorption spectroscopy (AAS), inductively coupled plasma emission or mass spectrometry (ICP-ES, ICP-MS), total reflection X-ray fluorimetry (TXRF) and anodic-stripping voltammetry (ASV) [3-5]. These methods offer good limits of detection and wide linear ranges, but they are time consuming, require high cost analytical instruments trained operating personals and high maintenance which is a financial burden to analytical laboratories. Furthermore, the required sample pretreatment and preparation time introduce systematic errors in the analysis. However, the development of fast, economical and portable devices for metal ion detection has grown tremendously over the past 10 years. Particularly, chemo-sensors, which offer the ability for both on-line and field monitoring, has attracted many industries in the detection of metal ions in water [6]. This has allowed for quick and continuous supervision monitoring of drinking or ground water and lentic or lotic watercourses. An ideal sensor should provide good sensitivity, high selectivity towards the target analyte, mathematical relationship of signal output to the amount of analyte, fast response time, good signal-to-noise ratio and longterm stability [7]. A variety of sensors have been developed, and these include DNAzymes sensors, optical sensors, electrochemical, colorimetric and fluorescent sensors [1-10] just to mention a few. This chapter aims to introduce the reader to the use of quantum dots (QDs) as metal ion sensors.

\subsection{Optical sensors}

A chemical sensor can be defined as 'a portable miniaturized analytical device, which can deliver real-time and on-line information in the presence of specific compounds or ions in complex samples' [11]. Chemical sensors can be categorized into electrochemical, optical, mass-sensitive and heat-sensitive, according to the types of transducer. Of these classifications, optical sensors have been the most widely used as contact-less detectors, counting or positioning of parts. An optical sensor device consists of the following components: (a) the recognition element, where specific interaction and identification of the analyte takes place; (b) the transducer element that converts the recognition process into a measurable optical signal; (c) an optical device (process unit) which consists of at least a light source and finally (d) a detector which detects and converts the change of optical properties and amplifies the signal into a unit readout. The optical properties measured can be absorbance, reflectance, luminescence, light polarization, Raman and others. Optical sensors have found many applications in various fields, including biomedical, clinical, environmental monitoring and process controlling [12-18]. They are an attractive analytical tool, whenever continuous monitoring and real-time information is desired. They can track sources of contamination in an industrial process, follow the formation and movement of environmental pollutants and can raise the alarm when a toxic species exceeds an expected level of exposure. For environmental analysis, 
single-use test strips for various ions, including heavy metals, are commercially available [19], which have their limitations in accuracy and reversibility. In recent years, activities applying optical sensors for the determination of heavy metals increased [20]. The most significant methods are the application of quenchable fluorophores or indicator dyes.

\subsection{Quantum dots}

Semiconductor quantum dots (QD) are nanocrystalline materials that confines the motion of the charge carriers in three spatial directions. These crystals are intermediate state of matter that display properties present in neither bulk nor molecular systems. The physical and electronic properties of QDs are strongly dependent on size (number of atoms). Their importance was recognized by the 2000 Nobel Prize in Physics awarded to Alferov and Kroemerin for their basic work on information and communication technology using the semiconductor heterostructures. QDs are generally made as binary semiconductor nanocrystals from groups II-VI (e.g. CdSe, $\mathrm{ZnS}$, etc.), groups IV-VI (e.g. PbTe, PbS) or groups III-V (e.g. InAs, InP) in the periodic table [21]. Moreover, QDs of $\mathrm{Si}, \mathrm{Ge}, \mathrm{Ag}$, also of carbon and graphene [22-24] and ternary QDs (from I-VI) have been reported $[25,26]$. QDs have received much attraction because of their stable, tunable, bright and narrow photoemission, high chemical and photo bleaching stability, processability and surface functionality and they offer many advantages in comparison with conventional fluorophore. Thus, a new generation of QDs based sensor containing the unique optical properties of QDs has been constructed for sensing molecules and ions in ultratrace level.

Colloidal synthesis of QDs widely employs a 'bottom-up' approach where the crystals are nucleated and grown from the precursor materials dissolved in a suitable solvent in the presence of a stabilizing agent. This approach was pioneered by L. Brus, an American scientist when he was at Bell laboratories, New Jersey, in the late 1970s and carried over by some of his post docs notably Moungi Bawendi and Paul Alivisatos. Because of its mild preparative conditions, this method is also called as a wet chemical route and has become popular among the scientists and industrialists for their usefulness in the biomedical and analytical fields. Different types of QDs such as alloyed QDs, core/shell QDs, impurity-doped QDs, polymerQD composites with desired size and desired functional group on the surface can be prepared by colloidal synthesis, which are difficult to obtain by the other methods.

High-quality colloidal quantum dot crystals can be prepared in organic medium. Organic QDs obtained by this method have good degree of monodispersity and high photoluminescence quantum efficiency. This method became familiar after the synthesis of CdSe QD by Murray et al. in 1993 [27, 28]. The precursors for CdSe QDs chosen by Murray were dimethylcadmium $\left(\mathrm{Me}_{2} \mathrm{Cd}\right)$ and TOPSe (Se dissolved in trioctylphosphine (TOP). The rapid injection of both the precursors together into the hot solution of trioctylphosphine oxide (TOPO) at $\sim 300^{\circ} \mathrm{C}$ produced yellow/orange CdSe nanocrystallites. However, the use of expensive and/or hazardous organic reagents, harsh reaction conditions, and hydrophobicity of the as-prepared QDs are some of the shortcomings of the organic synthetic routes. To make the QDs water soluble, hydrophobic nature of the QDs surface should be converted into hydrophilic nature by surface encapsulation or ligand exchange. Surface modification processes are tedious, involving 
multiple steps and usually produce materials with reduced optical properties compared to the parent organic materials $[29,30]$.

On the other hand, QDs can also be synthesized in aqueous medium directly. For example, in aqueous synthesis of thiol-stabilized CdTe QDs, $\mathrm{Cd}^{2+}$ dissolved in water medium would be reacted with a $\mathrm{HTe}^{-}$solution in the presence of water-soluble thiol ligands. Refluxing of the above mixture produces CdTe QDs dispersion. Rajh et al. used a thiol (3-mercapto-1,2propane-diol) as a stabilizing agent to prepare CdTe QD with 20\% photoluminescence quantum yield (PLQY) [31]. Afterwards, numerous thiols were investigated as stabilizing agents [32-34]. Compared with organic phase synthesis, aqueous synthesis involves less toxic precursors, inexpensive and produces excellent water-soluble and biocompatible products.

\subsection{Modifications for PL enhancement}

Most of the QDs, which are prepared in aqueous conditions, have low stability and low PLQY. As the surface of QDs is highly reactive, they have a high possibility to aggregate in the presence of heat, light, air or some ions. This may cause surface, which further reduced the PLQY. A number of techniques have been used to improve the particle stability, PL efficiency and biocompatibility of the QDs. These include photo-irradiation [35, 36], ultrasonic irradiation [37], doping with transition metals [38-40] and inorganic passivation [41]. Among them, inorganic passivation and doping techniques are the most widely investigated.

Doped semiconductor nanocrystals have been studied extensively in the past two decades since Bhargava et al. [42] reported on Mn-doped ZnS (Mn:ZnS). They stated that it could be possible to obtain efficient emission from the dopant centres even if the host nanocrystals were not of high quality. The PL of doped QDs is purely dopant-specific. Based on this, different colour-emitting (blue to red) QDs doped with metals $\left(\mathrm{Al}^{3+}\right)$, transition metals $\left(\mathrm{Cu}^{+}\right.$, $\left.\mathrm{Mn}^{2+}\right)$ and halides $\left(\mathrm{F}^{-}\right)$have been reported in the literature [43-45]. Doped nanocrystalline II-VI semiconductors incorporating rare earth (RE) ions such as $\mathrm{Tb}^{3+}, \mathrm{Eu}^{3+}$ and $\mathrm{Er}^{3+}$ have also been reported $[46,47]$. However, due to the dissimilar chemical properties (e.g. ionic radius, valence state) between the RE ion and host cation $\left(\mathrm{Cd}^{2+}, \mathrm{Zn}^{2+}\right)$, efficient doping of RE ions into II-VI semiconductor host is not favourable.

In contrast to RE ions, the chemical properties of $\mathrm{Mn}^{2+}$ are very similar to those of $\mathrm{Cd}^{2+}$ (or $\mathrm{Zn}^{2+}$ ); thus, incorporating $\mathrm{Mn}^{2+}$ into II-VI semiconductor host is much easier. Mn-doped semiconductors are potential luminescent and spintronic materials. The $\mathrm{Mn}^{2+}$ ion, used in many luminescent materials, has a $\mathrm{d}^{5}$ configuration. The $\mathrm{Mn}^{2+}$ ion exhibits a broad emission peak, whose position depends strongly on the host lattice due to changes in crystal field strength with host. The emission colour can vary from green to deep red, corresponding to a ${ }^{4} \mathrm{~T}_{1}-{ }^{6} \mathrm{~A}_{1}$ transition. Since this transition is spin-forbidden, the typical luminescent relaxation time of this emission is of the order of milliseconds.

Doping of the impurity in the host material can be carried out via nucleation-doping or growth-doping processes [48] as shown in Figure 1. In a former process, both host and dopant materials are subjected to nucleation at the same time followed by growth of the host 


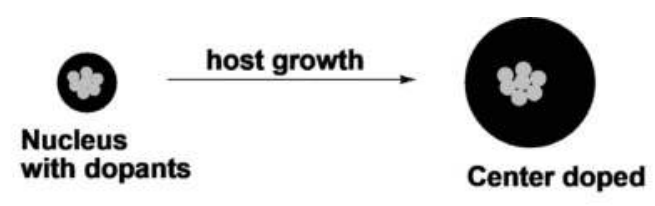

Nucleation doping

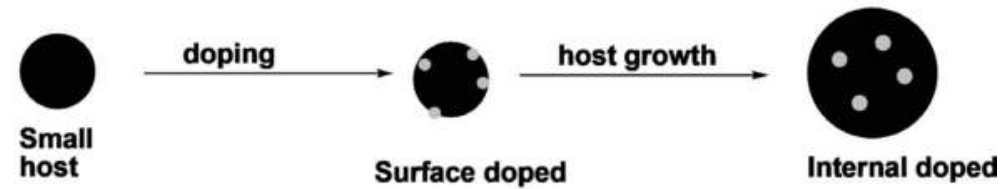

Growth doping

Figure 1. Schematic representations of nucleation- and growth-doping techniques.

material, whereas in the growth-doping process, the host material alone is nucleated and grow for certain time, followed by doping and re-growth of host material again.

\subsection{QDs as sensor}

QDs possess high surface-to-volume ratio and any change occurring at the surface can influence its surface-dependent properties; hence, luminescence of QDs is sensitive to surface states. Any species that interact directly with the QDs surface by physical or chemical means interferes with radiative recombination of the exciton leading to 'quenching' or enhancement of QDs fluorescence. Following this approach, QDs fluorescent probes can be designed by modifying their surface with suitable ligands so that they can selectively recognize the target analyte.

QD-based sensors are most frequently used to detect metal cations. Generally, metal ion quenches the QDs fluorescence via three different types of interaction: (i) by exchanging the metal cation of QDs, (ii) by displacing the capping ligand from the QDs surface and (iii) by binding with surface ligands [48]. The first two types are based on the competitive interaction between the analyte metal ion and the surface metal ion of QDs to bind with counterpart anion or surface-capping ligand, respectively, thus forming a stable lower solubility product. For instance, CuSe has a lower solubility than CdSe. As a result, surface $\mathrm{Cd}^{2+}$ ions in CdSe QDs can be easily exchanged by analyte $\mathrm{Cu}^{2+}$ ions to form $\mathrm{CuSe}$ particles on the surface of QDs. Similarly, in the case of glutathione (GSH)-capped CdSe QDs, $\mathrm{Pb}^{2+}$ ion binds with the thiol group of the capping GSH followed by displacement from QDs surface due to its higher binding affinity than $\mathrm{Cd}^{2+}$. However, the quenching mechanism in both cases is 
different. The electron and/or hole transfer from the CdSe to the CuSe energy levels (case i) is much faster than the process of fluorescence generation in the CdSe moiety, thus leading to quenching of QD fluorescence. The CuSe particles chemisorbed on CdSe QDs alters the bandgap energy level and crystal lattice. Hence, the shift in absorption, emission and X-ray diffractometry (XRD) patterns of the QDs after the interaction with the analyte metal ions is the indicative of this metal ion exchange process. By contrast, removing the capping ligand from the QDs surface by analyte metal ion (case ii) depassivates the QDs surface and creates drastic imperfections on the QDs surface, resulting in fluorescence quenching. In the ionbinding type (case iii), divalent analyte metal ions adsorb to the QD surface by electrostatic interaction with the capping carboxylic ligands, thus coordinating several QDs together, leading to the formation of closely packed QD aggregates. This causes a decrease in the luminescence intensity due to self-quenching mechanism. The quenching by this interaction is sensitive to $\mathrm{pH}$ and ionic strength of the medium. Under acidic conditions, quenching by metal ions is very low due to non-availability of carboxylate for the interaction with metal ions.

Apart from the metal ions, molecules such as phenolic compounds, $\mathrm{H}_{2} \mathrm{O}_{2}$ [49], 2,4,6-trinitrotoluene (TNT) [50,51] and glucose [52] can also be detected by fluorometric titration with QDs. The electron-accepting nature of phenolic compounds and TNT shuttled the electron from the conduction band to the valence band of the excited QDs, whereas $\mathrm{H}_{2} \mathrm{O}_{2}$ oxidizes the surface of QD and destroys its lattice structure resulting in the PL quenching. Glucose can be indirectly detected knowing that glucose can produce $\mathrm{H}_{2} \mathrm{O}_{2}$ on catalytic oxidation by glucose oxidase.

Most of the QDs that were investigated for fluorometric sensing are based on cadmium QDs; however, a major drawback for their application is the toxicity of cadmium ion. Less toxic particles like doped ZnS or ZnSe QDs may be interesting alternatives for biological imaging and other applications. $\mathrm{Mn}^{2+}$-doped $\mathrm{ZnS}$ quantum dots have been extensively investigated in various fields [53]. Fang et al. synthesized high-quality water-dispersible $\mathrm{Mn}^{2+}$-doped $\mathrm{ZnSe}$ core/ZnS shell (Mn:ZnSe/ZnS) nanocrystals directly in aqueous media with MPA as the capping ligand [54]. They observed that there was almost no dopant Mn emission in the Mn:ZnSe d-dots and bright Mn luminescence was observed after overcoating the ZnS shell around the Mn:ZnSe dots. In the present work, Mn-doped ZnSe (Mn:ZnSe) QDs have been synthesized by a wet chemical method using 3-mercaptopropionic acid (3-MPA) as capping agent and characterized by various analytical tools. The nucleation-doping method was adopted because it would form a structure similar to core-shell (MnSe/ZnSe) with a diffuse interface. The PL of the resulting QDs was examined in the presence of different metal ions to check its selective response.

\section{Methodology}

\subsection{Materials}

All chemicals were of analytical grade and were used without further purification. All solutions were prepared using doubly distilled water. $\mathrm{Zn}(\mathrm{CH} 3 \mathrm{COO})_{2} \cdot 2 \mathrm{H}_{2} \mathrm{O}\left(\mathrm{ZnAc}_{2}\right)$ and $\mathrm{Mn}$ 
$\left(\mathrm{CH}_{3} \mathrm{COO}\right)_{2} \bullet 4 \mathrm{H}_{2} \mathrm{O}\left(\mathrm{MnAc}_{2}\right)$ were obtained from Sisco Research Laboratories (SRL) Pvt. Ltd., Mumbai. Selenium powder (99.99\%) 3-mercaptopropionic acid and sodium borohydride $(\mathrm{NaBH} 4)$ were the AR reagents from Sigma Aldrich, Bangalore. Phosphate buffer at a $\mathrm{pH}$ of 7.0 was prepared according to literature. The chloride solutions of different metal ions were prepared at the concentration of $1 \times 10^{-4} \mathrm{M}$ using doubly distilled (DD) water.

\subsection{Synthesis of Mn-doped ZnSe QDs}

Aqueous colloidal solution of Mn:ZnSe QDs was synthesized using MPA as capping agent as depicted in Figure 2. Typically, $0.25 \mathrm{mmol}$ of selenium powder and $0.6 \mathrm{mmol}$ of $\mathrm{NaBH}_{4}$ were loaded in a round-bottomed flask containing $10 \mathrm{~mL}$ of $\mathrm{N}_{2}$-purged DD water. The reaction mixture was heated at $100^{\circ} \mathrm{C}$ for 15 min under $\mathrm{N}_{2}$ flow until the black selenium powder disappeared completely to give a clear colourless solution. On the other hand, $0.5 \mathrm{mmol}$ of $\mathrm{ZnAc}_{2}$ and 0.0015 or $0.003 \mathrm{mmol}$ of $\mathrm{MnAc}_{2}$ were dissolved in $25 \mathrm{~mL}$ of $\mathrm{DD}$ water followed by the addition of MPA. The $\mathrm{pH}$ of the reaction mixture was adjusted to 10.3 by adding $1 \mathrm{M} \mathrm{NaOH}$ solution and purged with $\mathrm{N}_{2}$ for $20 \mathrm{~min}$. Then, freshly prepared NaHSe solution was added followed by stirring at $50^{\circ} \mathrm{C}$ for $2 \mathrm{~h}$. The molar ratio of $\mathrm{Zn}$ to Se to MPA was set at 1:0.5:2, whereas $\mathrm{Zn}$ to $\mathrm{Mn}$ was 1:0.03 or 1:0.06. The as-synthesized nanoparticles were purified by precipitation with ethanol, followed by centrifugation and vacuum drying.

\subsection{Characterizations}

To investigate the optical, crystal structure and morphological properties of QDs, they were characterized by various analytical techniques. ultraviolet-visible (UV-vis) absorption spectra were recorded with a Shimadzu, (Model UV-1800) UV-visible spectrophotometer, Japan. The samples were dispersed in doubly distilled water and loaded in a 4.5-mL precleaned quartz cuvette with 1-cm optical path. The entire spectrum was scanned against the background spectrum of water. PL measurements of the samples were performed in a 4.5-mL quartz cuvette at ambient conditions on a Perkin Elmer LS5B spectrofluorimeter. For a given sample, the excitation wavelength was identified from the absorption spectrum

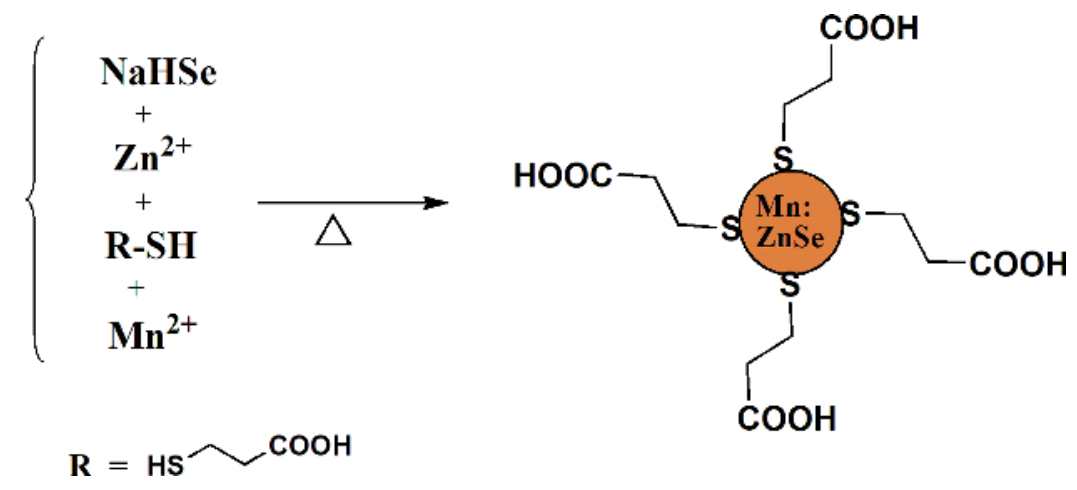

Figure 2. Synthesis of Mn-doped ZnSe QDs. 
and it was fixed to scan the emission wavelength. X-ray powder diffraction patterns of the samples were recorded at ambient conditions by using PANanalytical X'Pert PRO diffractometer with monochromatic $\mathrm{Cu}-\mathrm{K} \alpha 1$ radiation $(\lambda=1.5418 \AA), 2 \theta$ ranging from 10 to $80^{\circ}$ in steps of $0.017^{\circ} / \mathrm{s}$. The accelerating voltage was set at $40 \mathrm{kV}$ and the current flux was 30 $\mathrm{mA}$. Transmission electron microscopy (TEM) images of QDs were obtained from the FEI Tecnai G2 (T-30) instrument with the operating voltage of $250-300 \mathrm{kV}$. A small amount of sample for TEM analysis was ultra-sonicated in ethanol or water for a few minutes and then dropped on carbon-coated copper grids. The sample grid was then kept in vacuum desiccators prior to the analysis.

\subsection{Metal ion sensing}

Fluorescence sensitivity of the QDs towards different metal ions was carried out on a fluorescence microplate reader (Turners Biosystems-Modulus Microplate Multimode Reader-9300-010). Stock solutions of different metal ions $\left(\mathrm{Li}^{+}, \mathrm{Na}^{+}, \mathrm{K}^{+}, \mathrm{Mg}^{2+}, \mathrm{Ca}^{2+}, \mathrm{Ba}^{2+}, \mathrm{Al}^{3+}, \mathrm{Mn}^{2+}, \mathrm{Fe}^{2+}, \mathrm{Co}^{2+}, \mathrm{Ni}^{2+}\right.$, $\mathrm{Cu}^{2+}, \mathrm{Zn}^{2+}, \mathrm{Ag}^{+}, \mathrm{Cd}^{2+}, \mathrm{Hg}^{2+}$ and $\mathrm{Pb}^{2+}$ ) were prepared by dissolving their respective nitrate or chloride salts. Aqueous solution of QDs with the $\mathrm{OD}=0.1$ was prepared by diluting the stock solution of QDs. The solution of $50 \mu \mathrm{L}$ was dropped into each well of a 96-well plate followed by filling with different metal solutions to obtain the final volume of $200 \mu \mathrm{L}$. The excitation wavelength was selected according to the absorption spectrum of QDs and the relative fluorescence intensity was measured with the plate reader. The standard deviations were calculated from at least six measurements.

\section{Results and discussion}

\subsection{Absorption spectra}

Figure 3a shows the absorption spectra of 3 and 6\% Mn:ZnSe QDs along with undoped QDs. The Mn content is represented here as the mole \% against the host metal $(\mathrm{Zn})$ which is considered as $100 \%$ according to the experiment. Compared with bulk ZnSe having the bandgap of $\sim 2.8 \mathrm{eV}$, the absorption band edge of both doped and undoped QDs is blue-shifted (400-500 meV) indicating quantum confinement of the particles. Furthermore, the bandgap of Mn:ZnSe is blue-shifted with respect to undoped ZnSe QDs under the same experimental conditions, which reveal the formation of smaller-sized particles. This is consistent with the results by Mahamuni et al [55]. The synthesis process itself is affected by Mn additive. In addition, the spectrum of 3\% Mn:ZnSe QDs is slightly blue-shifted from that of 6\% Mn:ZnSe QDs. Because of lower $\mathrm{Mn} / \mathrm{Se}$ content, the former has relatively smaller-sized nuclei formed in the nucleation step which leads to a slight blue shift.

\subsection{PL spectra}

The PL spectra of the 3 and 6\% Mn:ZnSe QDs along with undoped QDs ( $\lambda$ exc $=365 \mathrm{~nm}$ ) are shown in Figure $3 \mathbf{b}$. The spectrum of undoped ZnSe QDs shows broad emission band centred 

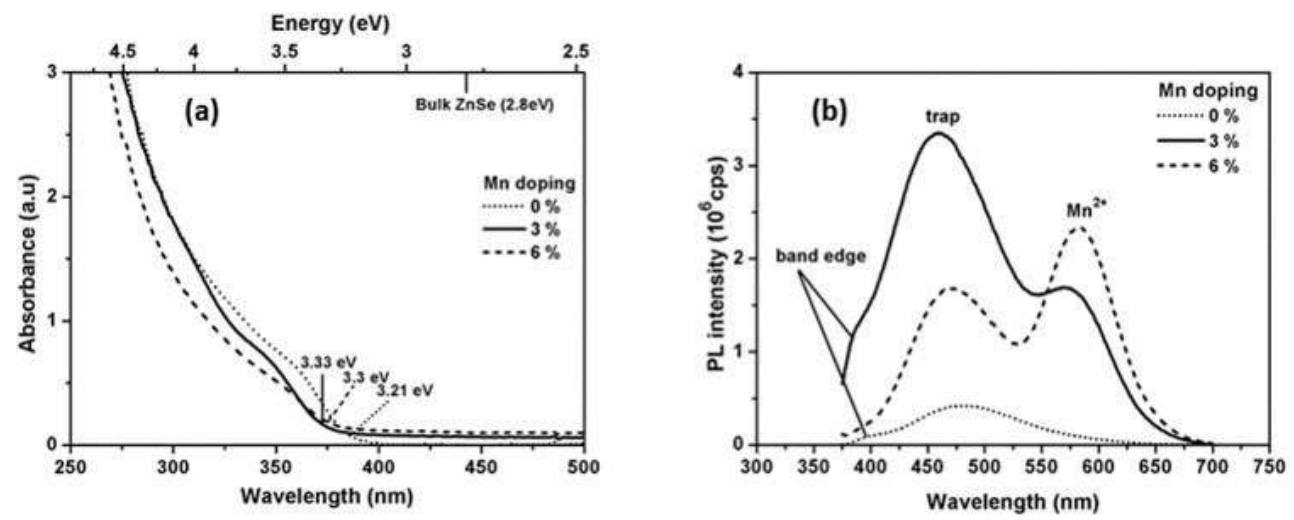

Figure 3. (a) Absorption and (b) PL spectra of undoped and Mn-doped ZnSe QDs.

at $480 \mathrm{~nm}$ and a small shoulder at $398 \mathrm{~nm}$ assigned to trap-state emission and band-edge emission, respectively. These two emissions appearing together are often observed in CdSe, ZnSe nanocrystals and their sulphur analogues. As discussed by Denzler et al [56], the bulk defects such as vacancies (Schottky defects) and interstitials (Frenkel defects) are the main source of trap states in the aqueous ZnSe QDs. With cubic zinc blende structure (by XRD), ZnSe usually has Schottky defects predominant over Frenkel defects. Therefore, the observed photoluminescence of QDs could be ascribed to a recombination of electrons at the selenium vacancy energy levels because of low $\mathrm{Se} / \mathrm{Zn}$ ratio synthetic conditions. There are many possible recombination paths through many trap-state emissions, each with different emission energy, causing the relatively wide emission peak. The PL spectrum of as-prepared ZnSe QDs has the full width at half maximum of about $110 \mathrm{~nm}$.

The PL spectrum of 3\% Mn:ZnSe QDs exhibits a new band at $579 \mathrm{~nm}$ in addition to trap- and band-edge emissions. The appearance of this new emission in the Mn-doped QDs is attributed to $\mathrm{Mn}^{2+}$-related ${ }^{4} \mathrm{~T}_{1} \rightarrow{ }^{6} \mathrm{~A}_{1}$ emission. This transition is spin-forbidden but is allowed because of crystal field effects [55]. Fang et al [54] notified that if the dopant ions are adsorbed on the surface of the host nanocrystal instead of being incorporated into its lattice, no dopant emission is observed and further the host emission is drastically quenched because a loosely adsorbed dopant ion can easily act as a surface trap that quenches the host PL [57]. However, in the present system, after doping with 3\% Mn, the luminescence of host emission is enhanced by 7.7 times along with the appearance of Mn emission. This indicates the successful incorporation of $\mathrm{Mn}^{2+}$ in the host ZnSe lattice. The enhancement of host PL is mainly attributed to the reduction of non-radiative energy centres by $\mathrm{Mn}^{2+}$ doping. Table 1 shows the PL-integrated intensity ratios calculated from the spectra.

With further increase in the Mn concentration from 3 to $6 \%$, the resultant doped QDs show increased Mn emission followed by subtle red-shift indicating the increase in Mn content in host. However, the host-trap emission is decreased and no band-edge emission is significantly observed. Furthermore, instead of the expected two-fold increase in the Mn emission 


\begin{tabular}{|c|c|c|c|c|c|c|c|c|c|}
\hline \multicolumn{10}{|c|}{ PL-integrated intensity ratios of Mn:ZnSe QDs } \\
\hline Mn doping & Band-edge & & Trap & & Mn & & Cumulative & $\begin{array}{l}\text { Trap/Band } \\
\text { edge }\end{array}$ & Trap/Mn \\
\hline & Peak (nm) & $\mathrm{I} / \mathrm{I}_{0 \%}$ & Peak (nm) & $\mathrm{I} / \mathrm{I}_{0 \%}$ & Peak (nm) & $\mathrm{I} / \mathrm{I}_{3 \%}$ & $\mathrm{I} / \mathrm{I}_{0 \%}$ & $I_{t} / I_{b}$ & $I_{t} / I_{M n}$ \\
\hline $0 \%$ & 397 & 1.0 & 480 & 1.0 & - & - & 1.0 & 82.2 & - \\
\hline $3 \%$ & 386 & 6.7 & 461 & 7.7 & 579 & 1.0 & 10.1 & 95.7 & 3.2 \\
\hline $6 \%$ & - & - & 473 & 3.1 & 584 & 1.4 & 6.5 & - & 0.9 \\
\hline
\end{tabular}

${ }^{*} \mathrm{t}=$ trap, $\mathrm{b}=$ band edge. (PL peak position and the corresponding integrated intensity are obtained by Gaussian fitting.)

Table 1. PL properties of undoped and Mn-doped ZnSe QDs.

(from 3\% doped), only 1.4-fold increase is observed. The reason might be the combination of the following three processes: (i) non-radiative energy transfer between neighbouring $\mathrm{Mn}^{2+}$-dopant ions which quenches Mn emission [58], (ii) adsorption of some Mn ions on the surface instead of incorporation into the host lattice which quenches both host and Mn emission and (iii) initially formed larger MnSe. As the environment of the doping ions in larger nuclei is not as uniform as that in smaller ones, the PL emission performance of doped QDs with larger nuclei will not be satisfactory as those with smaller nuclei [57]. Overall, the PL peak position of the host is in tune with the corresponding band-edge absorption. The 3\% doped sample has the highest emission peak intensity, followed by the $6 \%$ and then the $0 \%$ sample. The above results are interesting that the ratio of the dual-colour emissions (blue and orange) of the Mn-doped ZnSe QDs could be controlled by tuning the Mn-doping levels in the QDs.

\subsection{Structural and morphological analysis}

X-ray diffraction pattern of the 3\% Mn:ZnSe QDs is presented in Figure 4. Broad diffraction peaks are observed and are attributed to the nanocrystalline nature of the material. The XRD peaks are close to the characteristic peaks corresponding to the (111), (220) and (311) planes of cubic zinc blende ZnSe. Cubic structures are often obtained in the low-temperature aqueous synthesis of ZnSe QDs. This indicates that the incorporation of $\mathrm{Mn}^{2+}$ into the host $\mathrm{ZnSe}$ does not bring any obvious change in the crystal lattice and the structure [37, 54]. TEM image of the 3\% Mn:ZnSe QDs (Figure 5a) shows that QDs are spherical in nature with the average diameter of $\sim 4 \mathrm{~nm}$. Some of the aggregates of the particles are also seen. The energy-dispersive $\mathrm{X}$-ray spectroscopy (EDS) spectrum (Figure $5 \mathbf{b}$ ) confirms the presence of $\mathrm{Mn}, \mathrm{Zn}$ and Se and the purity of the sample.

\subsection{Effect of metal ions on the PL intensity}

The fluorescence titrations of the $3 \% \mathrm{Mn}: \mathrm{ZnSe}$ QDs (PL, $\lambda_{\max }=461 \mathrm{~nm}$ ) with various metal ions are shown in Figure 6a. The fluorescence intensity $(\mathrm{F})$ is measured with excitation at $365 \mathrm{~nm}$. The PL of blank QDs is used as a control $\left(\mathrm{F}_{0}\right)$. From the figure, it is observed that QDs show maximum response to mercury ions $\left(\mathrm{Hg}^{2+}\right)$, little response to $\mathrm{Ni}^{2+}, \mathrm{Cu}^{2+}$ and $\mathrm{Pb}^{2+}$ ions and 


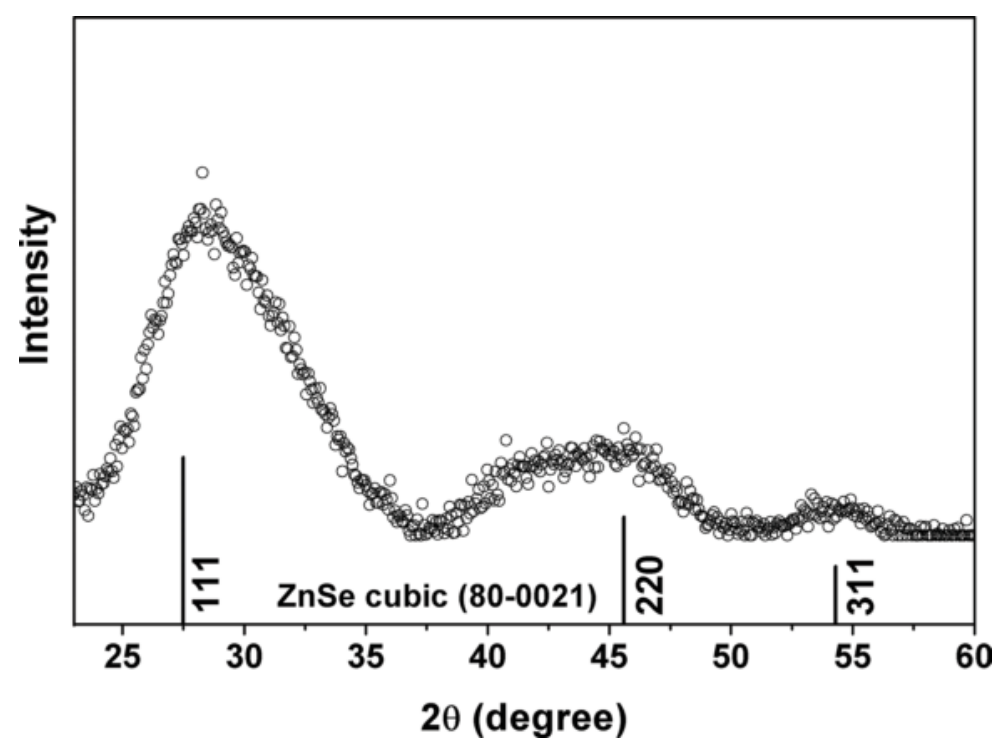

Figure 4. XRD patterns of 3\% Mn-doped ZnSe QDs.
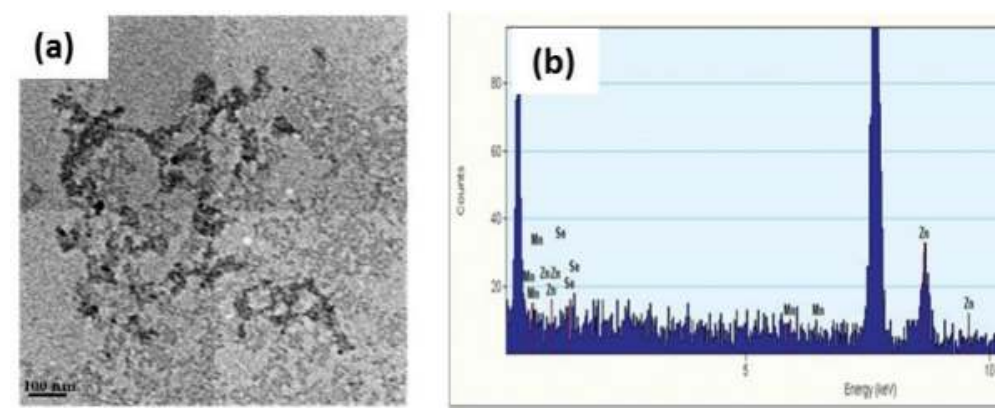

Figure 5. (a) TEM image and (b) EDS of 3\% Mn-doped ZnSe QDs.

almost no response to other metal ions. About $74 \%$ of the PL intensity is quenched after the addition of $30 \mu \mathrm{M} \mathrm{L}^{-1}$ of $\mathrm{Hg}^{2+}$ ions.

The influence of $\left[\mathrm{Hg}^{2+}\right]$ ion on the QDs fluorescence was studied. Figure $6 \mathbf{b}$ shows the quenching behaviour of $\mathrm{Hg}^{2+}$ ions on the PL intensity of QDs. The PL intensity is quenched drastically and then slightly with the increase in $\left[\mathrm{Hg}^{2+}\right]$ ions. The fluorescence quenching with respect to the concentration of quencher was analysed using the Stern-Volmer equation. The plot of $\mathrm{F}_{0} / \mathrm{F}$ versus $\left[\mathrm{Hg}^{2+}\right]$ as shown in Figure 6c exhibits a good linear relationship up to 30 $\mu \mathrm{M} \mathrm{L}^{-1}$ with a correlation coefficient $\mathrm{R}^{2}=0.9918$. The limit of detection (LOD) was calculated according to the following equation: 

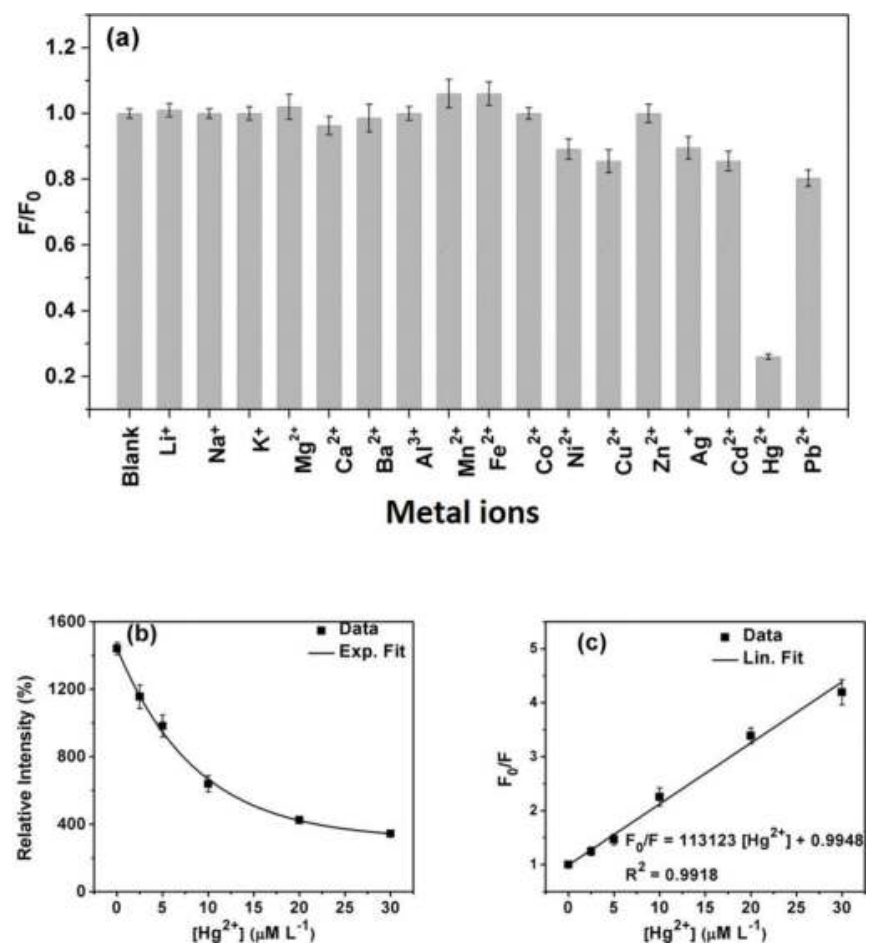

Figure 6. (a) Effect of metal ions $\left(30 \mu \mathrm{M} \mathrm{L}^{-1}\right)$ on the PL intensity of $3 \%$ Mn-doped ZnSe QDs at pH 10.8. (b) The plot of PL intensity of 3\% Mn-doped ZnSe QDs versus $\left[\mathrm{Hg}^{2+}\right]$ ions and (c) the corresponding Stern-Volmer relationship.

$$
\mathrm{LOD}=\frac{3 \mathrm{~S}_{0}}{\mathrm{~K}_{\mathrm{sv}}}
$$

where 3 is the signal-to-noise ratio, $S_{0}$ is the standard deviation of blank measurements $(n=6)$ and $\mathrm{K}_{\mathrm{sv}}$ is the slope of calibration graph. LOD of the present probe towards $\left[\mathrm{Hg}^{2+}\right]$ under the experimental conditions is found to be $6.63 \times 10^{-7} \mathrm{M} \mathrm{L}^{-1}$.

\subsection{Mechanism of sensing by quenching}

The fluorescence quenching can be explained in terms of strong binding of quencher metal ions $\left(\mathrm{Hg}^{2+}\right)$ on the surface of QDs. It is known that the solubility $\mathrm{HgSe}\left(\mathrm{K}_{\mathrm{sp}}=2 \times 10^{-53}\right)$ is extremely lower than that of $\mathrm{ZnSe}\left(\mathrm{K}_{\mathrm{sp}}=3.6 \times 10^{-26}\right)$. The low solubility product is always preferred in a solution and hence the quencher metal ions $\left(\mathrm{Hg}^{2+}\right)$ displace the $\mathrm{Zn}$ on the surface of QDs and form a lower solubility product (HgSe) which deposit on the surface of the QDs

$$
\mathrm{Zn}_{\mathrm{m}} \mathrm{Se}_{\mathrm{n}}+\mathrm{xHg}^{2+} \rightarrow \mathrm{Zn}_{\mathrm{m}-\mathrm{x}} \mathrm{Hg}_{\mathrm{x}} \mathrm{Se}_{\mathrm{n}}+\mathrm{xZn^{2+ }}
$$

The formed particles of HgSe, both isolated and aggregated, can quench the luminescence of QDs by facilitating non-radiative annihilation of charge carriers, which act as electron-hole 

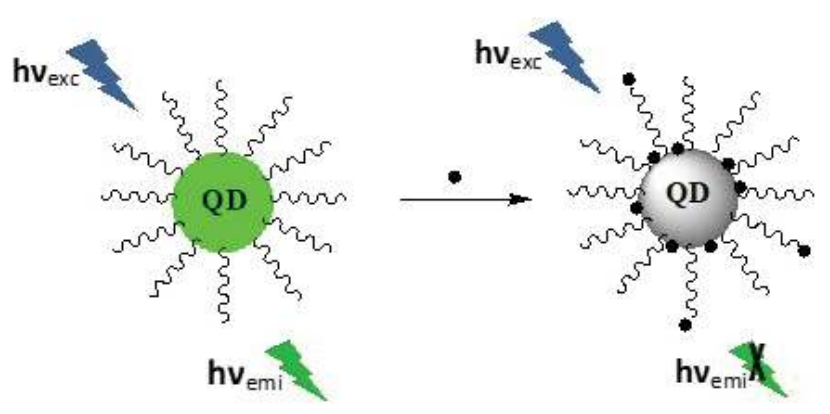

nur Ligand

- Metal ion

Figure 7. Schematic representation of fluorescence quenching of QDs by metal ions.

recombination centres [59]. However, mere $\mathrm{K}_{\mathrm{sp}}$ values are not the sole factors in fluorescence quenching. In addition, surface ligands have profound effects on the fluorescence response of QDs to metal ions [60]. They play a critical role in metallic ion selectivity [61]. Also, the quenching of luminescence of the QDs can occur partly through ion binding followed by photoinduced electron transfer process from the thiol ligand to $\mathrm{Hg}^{2+}$ ions on the surface of QDs. The schematic representation of fluorescence quenching is shown in Figure 7. Theoretical calculation and further study are in progress to gain an insight into the mechanism of fluorescence quenching.

\section{Conclusions}

In summary, water-soluble MPA-capped Mn-doped ZnSe QDs were synthesized using nucleation-doping method. The absorption spectra of the as-synthesized QDs were blue-shifted in relation to the bulk counterparts due to quantum confinement. The QDs exhibited dual-colour emission (blue and orange). The intensity ratio of the dual-colour emission can be varied by tuning the Mn-doping percentage. It was found that $3 \% \mathrm{Mn}$ doping in the ZnSe crystal lattice increases the fluorescence (blue) of ZnSe QDs by 10-fold due to the reduction of non-radiative energy centres. Furthermore, $\mathrm{Mn}^{2+}$-related ${ }^{4} \mathrm{~T}_{1} \rightarrow{ }^{6} \mathrm{~A}_{1}$ (orange) emission characteristic of Mn doped in the ZnSe host was observed. The as-prepared QDs exhibited a cubic crystal structure according to XRD patterns. TEM images showed aggregates of tiny spherical particles with the average diameter of $\sim 4 \mathrm{~nm}$. The as-prepared $\mathrm{Mn}_{3 \%}: \mathrm{ZnSe}$ QDs were treated with different metal ions and were found to be highly selective to $\mathrm{Hg}^{2+}$ ions and exhibit pleasing LOD. The possible mechanism of sensing by quenching was also proposed. These studies on metal ion sensing by fluorescent QDs have demonstrated their potential as chemical sensor which can be developed for industrial applications. 


\section{Author details}

Sundararajan Parani ${ }^{1,2}$, Ncediwe Tsolekile ${ }^{1,2,3}$, Bambesiwe M.M. May ${ }^{1,2}$, Kannaiyan Pandian ${ }^{4}$ and Oluwatobi S. Oluwafemi ${ }^{1,2 *}$

*Address all correspondence to: oluwafemi.oluwatobi@gmail.com

1 Department of Applied Chemistry, University of Johannesburg, Doornfontein, Johannesburg, South Africa

2 Centre for Nanomaterials Science Research, University of Johannesburg, Johannesburg, South Africa

3 Department of Chemistry, Cape Peninsula University of Technology, Cape Town, South Africa

4 Department of Inorganic Chemistry, University of Madras, Chennai, India

\section{References}

[1] McGhee CE, Yong Loh K, Lu Y. DNAzyme sensors for detection of metal ions in the environment and imaging them in living cells. Current Opinion in Biotechnology [Internet]. 2017;45:191-201 Available from: http://dx.doi.org/10.1016/j.copbio.2017.03.002

[2] Barone C, Bolzoni L, Caruso G, Montanari A, Parisi S, Steinka I. Food Packaging Hygiene. 2015. Available from: http://link.springer.com/10.1007/978-3-319-14827-4

[3] Zhong WS, Ren T, Zhao LJ. Determination of Pb (Lead), Cd (Cadmium), Cr (Chromium), $\mathrm{Cu}$ (Copper), and Ni (Nickel) in Chinese tea with high-resolution continuum source graphite furnace atomic absorption spectrometry. Journal of Food and Drug Analysis [Internet]. 2016;24(1):46-55 Available from: http://dx.doi.org/10.1016/j. jfda.2015.04.010

[4] McComb JQ, Rogers C, Han FX, Tchounwou PB. Rapid screening of heavy metals and trace elements in environmental samples using portable X-ray fluorescence spectrometer: A comparative study. Water, Air, \& Soil Pollution [Internet]. 2014;225(12):2169 Available from: http://link.springer.com/10.1007/s11270-014-2169-5

[5] Barón-Jaimez J, Joya MR, Barba-Ortega J. Anodic stripping voltammetry - ASV for determination of heavy metals. Journal of Physics: Conference Series [Internet]. 2013;466:12023 Available from: http://stacks.iop.org/1742-6596/466/i=1/a=012023?key=crossref.8f5785f72 a4a87ee677967514ca4e165

[6] Verma R, Gupta BD. Fiber optic SPR sensor for the detection of 3-pyridinecarboxamide (vitamin B3) using molecularly imprinted hydrogel. Sensors and Actuators B: Chemical [Internet]. 2013;177:279-285 Available from: http://dx.doi.org/10.1016/j.snb.2012.10.135 
[7] Deng G. Principles of chemical and biological sensors. Materials and Manufacturing Processes [Internet]. 1999;14(4):623-625 Available from: http://www.tandfonline.com/ doi/abs/10.1080/10426919908907570

[8] Neupane LN, Oh ET, Park HJ, Lee KH. Selective and sensitive detection of heavy metal ions in $100 \%$ aqueous solution and cells with a fluorescence chemosensor based on peptide using aggregation-induced emission. Analytical Chemistry. 2016;88(6):3333-3340

[9] Verma R, Gupta BD. Detection of heavy metal ions in contaminated water by surface plasmon resonance based optical fibre sensor using conducting polymer and chitosan. Food Chemistry [Internet]. 2015;166:568-575 Available from: http://dx.doi.org/10.1016/j. foodchem.2014.06.045

[10] Vázquez-González M, Carrillo-Carrion C. Analytical strategies based on quantum dots for heavy metal ions detection. Journal of Biomedical Optics [Internet]. 2014;19(10):101503 Available from: http://biomedicaloptics.spiedigitallibrary.org/article. aspx?doi=10.1117/1.JBO.19.10.101503

[11] Chen Y, Li S, Huang L, Pan D. Low-cost and gram-scale synthesis of water-soluble $\mathrm{Cu}-\mathrm{In}-\mathrm{S} / \mathrm{ZnS}$ core/shell quantum dots in an electric pressure cooker. Nanoscale. 2014;6:1295-1298 Available from: http://pubs.rsc.org/en/Content/ArticleLanding/2014/ NR/C3NR05014A\#!divAbstract

[12] Szulczyński B, Gębicki J. Currently commercially available chemical sensors employed for detection of volatile organic compounds in outdoor and indoor air. Environments [Internet]. 2017;4(1):21 Available from: http://www.mdpi.com/2076-3298/4/1/21

[13] Mehrotra P. Biosensors and their applications-A review. Journal of Oral Biology and Craniofacial Research [Internet]. 2016;6(2):153-159 Available from: http://dx.doi. org/10.1016/j.jobcr.2015.12.002

[14] Ramasamy R, Gopal N, Kuzhandaivelu V, Murugaiyan S. Biosensors in clinical chemistry: An overview. Advanced Biomedical Research [Internet]. 2014;3(1):67 Available from: http://www.advbiores.net/text.asp?2014/3/1/67/125848

[15] Yeh P, Yeh N, Lee CH, Ding TJ. Applications of LEDs in optical sensors and chemical sensing device for detection of biochemicals, heavy metals, and environmental nutrients. Renewable \& Sustainable Energy Reviews [Internet]. 2017;75:461-468 Available from: http://dx.doi.org/10.1016/j.rser.2016.11.011

[16] Long F, Zhu A, Shi H. Recent advances in optical biosensors for environmental monitoring and early warning. Sensors (Basel). 2013;13(10):13928-13948

[17] Weichert F, Bachmann D, Rudak B, Fisseler D. Analysis of the accuracy and robustness of the Leap Motion Controller. Sensors (Switzerland). 2013;13(5):6380-6393

[18] Mathews CB, Libish TM, Kaushalkumar B, Vivek V, Prabhu R, Radhakrishnan P. A fiber optic biosensor for the detection of cholesterol levels based on chitosan coated long period grating. Optoelectronics Letters. 2016;12(1):23-26 
[19] Daou TJ, Pourroy G, Bégin-Colin S, Grenèche JM, Ulhaq-Bouillet C, Legaré P, et al. Hydrothermal synthesis of monodisperse magnetite nanoparticles. Chemistry of Materials. 2006;18(18):4399-4404

[20] Oehme I, Wolfbeis OS. Optical sensors for determination of heavy metal ions. Microchimica Acta. 1997;126:177-192

[21] Oluwafemi SO, Revaprasadu N, Ramirez AJ. A novel one-pot route for the synthesis of water-soluble cadmium selenide nanoparticles. Journal of Crystal Growth. 2008;310 (13):3230-3234

[22] Wang R, Lu K-Q, Tang Z-R, Xu Y-J. Recent progress on carbon quantum dots: Synthesis, properties and applications in photocatalysis. Journal of Materials Chemistry A [Internet]. 2017;5:3717-3734 Available from: http://pubs.rsc.org/en/Content/ArticleLanding/2017/ TA/C6TA08660H

[23] Kang X, Yang Y, Huang L, Tao Y, Wang L, Pan D. Large-scale synthesis of water-soluble $\mathrm{CuInSe}_{2} / \mathrm{ZnS}$ and $\mathrm{AgInSe}_{2} / \mathrm{ZnS}$ core/shell quantum dots. Green Chemistry [Internet]. 2015;17(8):4482-4488 Available from: http://dx.doi.org/10.1039/C5GC00908A

[24] Raeyani D, Shojaei S, Kandjani SA, Wlodarski W. Synthesizing graphene quantum dots for gas sensing applications. Procedia Engineering [Internet]. 2016;168:1312-1316 Available from: http://dx.doi.org/10.1016/j.proeng.2016.11.356

[25] Chen Y, Li S, Huang L, Pan D. Green and facile synthesis of water-soluble Cu-In-S/ZnS core/shell quantum dots. Inorganic Chemistry. 2013;52(14):7819-7821

[26] Chuang P, Lin CC, Liu R. Emission-tunable $\mathrm{CuInS}_{2} / \mathrm{ZnS}$ quantum dots: Structure, optical properties, and application in white light-emitting diodes with high color rendering index. Applied Materials and Interfaces. 2014;6(17):15379-15387

[27] Malik P, Singh J, Kakkar R. A review on CdSe quantum dots in sensing. Advanced Materials Letters. 2014;5(11):612-628

[28] Murray CB, Norris DJ, Bawendi MG. Synthesis and characterization of nearly monodisperse $\mathrm{CdE}$ ( $\mathrm{E}$ = sulfur, selenium, tellurium) semiconductor nanocrystallites. Journal of the American Chemical Society [Internet]. 1993;115(19):8706-8715 Available from: http:// pubs.acs.org/doi/abs/10.1021/ja00072a025

[29] Draaisma GJJ, Reardon D, Schenning APHJ, Meskers SCJ, Bastiaansen CWM. Ligand exchange as a tool to improve quantum dot miscibility in polymer composite layers used as luminescent down-shifting layers for photovoltaic applications. Journal of Materials Chemistry C [Internet]. 2016;4(24):5747-5754 Available from: http://xlink.rsc. org/?DOI=C6TC01261B

[30] Karakoti AS, Shukla R, Shanker R, Singh S. Surface functionalization of quantum dots for biological applications. Advances in Colloid and Interface Science [Internet]. 2015;215:28-45 Available from: http://dx.doi.org/10.1016/j.cis.2014.11.004 
[31] Rajh T, Micic OI, Nozik AJ. Synthesis and characterization of surface-modified colloidal cadmium telluride quantum dots. The Journal of Physical Chemistry. 1993;97:1199912003 Available from: http://pubs.acs.org/doi/abs/10.1021/j100148a026

[32] Oluwafemi OS, Daramola OA, Ncapayi V. A facile green synthesis of type II water soluble CdTe/CdS core shell nanoparticles. Materials Letters [Internet]. 2014;133:9-13 Available from: http://www.sciencedirect.com/science/article/pii/S0167577X14012014

[33] Ncapayi V, Parani S, Songca SP, Kodama T, Oluwafemi OS. Simple green synthesis of amino acid functionalised $\mathrm{CdTe} / \mathrm{CdSe} / \mathrm{ZnSe}$ core-multi shell with improved cell viability for cellular imaging. Materials Letters [Internet]. 2017;189:168-171 Available from: http:// dx.doi.org/10.1016/j.matlet.2016.12.006

[34] Parani S, Tsolekile N, Pandian K, Oluwafemi OS. Thiolated selenium as a new precursor for the aqueous synthesis of CdSe/CdS core/shell quantum dots. Journal of Materials Science: Materials in Electronics. 2017;28:11151. DOI: 10.1007/s10854-017-6902-x

[35] Krivenkov V, Tretyachenko A, Samokhvalov PS, Chistyakov AA, Nabiev I. Controllable photo-brightening/photo-darkening of semiconductor quantum dots under laser irradiation. Proceedings of SPIE [Internet]. 2016;9884:98843L Available from: http://proceedings.spiedigitallibrary.org/proceeding.aspx?doi=10.1117/12.2228815

[36] Luo C, Li B, Peng H, Tang X, Wang Y, Travas-Sejdic J. The effect of photo-irradiation on the optical properties of thiol-capped CdTe quantum dots. Journal of Nanoscience and Nanotechnology [Internet]. 2012;12(4):2998-3005 Available from: http://www.ncbi.nlm. nih.gov/pubmed/22849057

[37] Wang X, Kong X, Yu Y, Sun Y, Zhang H. Effect of annealing on upconversion luminescence of $\mathrm{ZnO}: \mathrm{Er} 3+$ nanocrystals and high thermal sensitivity. The Journal of Physical Chemistry C [Internet]. 2007;111(41):15119-15124 Available from: http://pubs.acs.org/ doi/abs/10.1021/jp0686689

[38] Saleh R, Djaja NF. Transition-metal-doped ZnO nanoparticles: Synthesis, characterization and photocatalytic activity under UV light. Spectrochimica Acta Part A: Molecular and Biomolecular Spectroscopy [Internet]. 2014;130:581-590 Available from: http:// dx.doi.org/10.1016/j.saa.2014.03.089

[39] Schelonka D, Tolasz J, Štengl V. Doping of zinc oxide with selected first row transition metals for photocatalytic applications. Photochemistry and Photobiology. 2015;91(5):1071-1077

[40] Aleksandrova OA, Mazing DS, Musikhin SF, Nikiforova AV. Synthesis of transition metal doped zinc selenide nanoparticles for bioimaging. Photonics North. 2015:1 doi: 10.1109/PN.2015.7292477

[41] Mathew S, Bhardwaj BS, Saran AD, Radhakrishnan P, Nampoori VPN, Vallabhan CPG, et al. Effect of ZnS shell on optical properties of CdSe-ZnS core-shell quantum dots. Optical Materials (Amsterdam). 2015;39:46-51 
[42] Bhargava RN, Gallagher D, Hong X, Nurmikko A. Optical properties of manganesedoped of ZnS. Physical Review Letters. 1994;72(3):1-4

[43] Rajesh C, Phadnis C, Sonawane KG, Mahamuni S. Generation of white light from co-doped ( $\mathrm{Cu}$ and $\mathrm{Mn})$ ZnSe QDs. Journal of Experimental Nanoscience [Internet]. 2014;10(14):1082-1092 Available from: http://www.tandfonline.com/doi/abs/10.1080/174 58080.2014 .964340

[44] Rajesh C, Phadnis CV, Sonawane KG, Mahamuni S. Synthesis and optical properties of coppper-doped ZnSe quantum dots. Physica Scripta [Internet]. 2015;90(1):15803 Available from: $\mathrm{http}: / /$ stacks.iop.org/1402-4896/90/i=1/a=015803?key=crossref.d67be3a40db02be aa895c20d8952b09f

[45] Aphesteguy JC, Kurlyandskaya GV, de Celis JP, Safronov AP, Schegoleva NN. Magnetite nanoparticles prepared by co-precipitation method in different conditions. Materials Chemistry and Physics [Internet]. 2015;161:243-249. Available from: http://www.sciencedirect.com/science/article/pii/S0254058415301073

[46] Wang Y, Liang X, Liu E, Hu X, Fan J. Incorporation of lanthanide (Eu(3+)) ions in ZnS semiconductor quantum dots with a trapped-dopant model and their photoluminescence spectroscopy study. Nanotechnology [Internet]. 2015;26(37):375601 Available from: http://apps.webofknowledge.com/full_record.do?product=UA\&search_mode=G eneralSearch\&qid=23\&SID=V176ODJebApDXuKoNAp\&page=1\&doc=6\&cacheurlFrom RightClick=no

[47] Sureshkumar S, Jothimani B, Sridhar TM, Venkatachalapathy B. Synthesis and characterization of gadolinium-doped $\mathrm{ZnSe}$ quantum dots for fluorescence imaging of cancer cells. RSC Advances [Internet]. 2016;6(19):16081-16086 Available from: http://xlink.rsc. org/?DOI=C5RA18773G

[48] Guria AK, Pradhan N. Doped or not doped: Ionic impurities for influencing the phase and growth of semiconductor nanocrystals. Chemistry Materials. 2016;28(15):5224-5237

[49] $\mathrm{Li} \mathrm{Y,} \mathrm{Li} \mathrm{B,} \mathrm{Zhang} \mathrm{J.} \mathrm{H2O2-} \mathrm{and} \mathrm{pH-sensitive} \mathrm{CdTe} \mathrm{quantum} \mathrm{dots} \mathrm{as} \mathrm{fluorescence} \mathrm{probes}$ for the detection of glucose. Luminescence. 2013;28(5):667-672

[50] Shamirian A, Ghai A, Snee PT. QD-based FRET probes at a glance. Sensors. 2015;15(6): 13028-13051

[51] Xu S, Lu H, Li J, Song X, Wang A, Chen L, et al. Dummy molecularly imprinted polymers-capped CdTe quantum dots for the fluorescent sensing of 2,4,6-trinitrotoluene. ACS Applied Materials \& Interfaces. 2013;5(16):8146-8154

[52] Ding L, Zhang B, Xu C, Huang J, Xia Z. Fluorescent glucose sensing using CdTe/CdS quantum dots-glucose oxidase complex. Analytical Methods [Internet]. 2016;8(14):29672970 Available from: http://xlink.rsc.org/?DOI=C5AY03205A

[53] Subha R, Nalla V, Yu JH, Jun SW, Shin K, Hyeon T, et al. Efficient photoluminescence of $\mathrm{Mn}^{2+}$-doped $\mathrm{ZnS}$ quantum dots excited by two-photon absorption in near-infrared window II. Journal of Physical Chemistry C. 2013;117(40):20905-20911 
[54] Fang Z, Wu P, Zhong X, Yang Y. Synthesis of highly luminescent Mn:ZnSe/ZnS nanocrystals in aqueous media. Nanotechnology. 2010;21(30):305604

[55] Mahamuni S, Lad AD, Patole S. Photoluminescence properties of manganese-doped zinc selenide quantum dots. Journal of Physical Chemistry C. 2008;112(7):2271-2277

[56] Denzler D, Olschewski M, Sattler K. Luminescence studies of localized gap states in colloidal ZnS nanocrystals. Journal of Applied Physics [Internet]. 1998;84(5):2841-2845 Available from: http://aip.scitation.org/doi/10.1063/1.368425

[57] Pradhan N, Battaglia DM, Liu Y, Peng X. Efficient, stable, small, and water-soluble doped ZnSe nanocrystal emitters as non-cadmium biomedical labels. Nano Letters. 2007;7(2):312-317

[58] Sooklal K, Cullum BS, Angel SM, Murphy CJ. Photophysical properties of ZNS nanoclusters with spatially localized $\mathrm{Mn}^{2+}$. Journal of Physical Chemistry [Internet]. 1996;100(11):4551-4555 Available from: http://pubs.acs.org/doi/abs/10.1021/jp952377a

[59] Isarov AV, Chrysochoos J. Optical and photochemical properties of nonstoichiometric cadmium sulfide nanoparticles: Surface modification with copper(II) ions. Langmuir. 1997;13(12):3142-3149

[60] Chen Y, Rosenzweig Z. Luminescent CdS quantum dots as selective ion probes. Analytical Chemistry. 2002;74(19):5132-5138

[61] Gattás-Asfura KM, Leblanc RM. Peptide-coated CdS quantum dots for the optical detection of copper(II) and silver(I). Chemical Communications [Internet]. 2003;2003(21):26842685 Available from: http://www.ncbi.nlm.nih.gov/pubmed/14649810 
